\title{
Complementary Food Hygiene Practice among Mothers or Caregivers in Bale Zone, Southeast Ethiopia: A Community Based Cross-Sectional Study
}

Shumi Bedada ( $\sim$ shumib17@gmail.com )

Bale Zone Health Department https://orcid.org/0000-0003-3875-0264

Mekonnen Tegegne

Madda Walabu University Goba Referral Hospital

Tomas Benti

Madda Walabu University Goba Referral Hospital

\section{Research Article}

Keywords: Complementary food, Food hygiene, Handwashing, Mothers or Caregivers, Practice

Posted Date: June 22nd, 2021

DOI: https://doi.org/10.21203/rs.3.rs-633101/v2

License: (c) This work is licensed under a Creative Commons Attribution 4.0 International License.

Read Full License 


\section{Abstract}

Introduction: Foodborne diseases are a major public health problem which predominantly affects infants and young children. Appropriate complementary food hygiene practice is very important to reduce the prevalence of foodborne illnesses. However, the information regarding this practice is not available and assessed before. Thus, the study was aimed to assess complementary food hygiene practices among mothers of children aged 6-23 months.

Methods: A community based cross-sectional study was conducted in Robe town on a samples of 517 mother-child pairs, which were selected by using systematic sampling technique. Data was collected using pre-tested and structured questionnaire, and each variable was described in the frequency and percentage. Bivariable and multivariable logistic regression analyses were used to identify factors associated with complementary food hygiene practice. Odds ratios with $95 \%$ confidence interval were used to assess levels of significance.

Result: The result indicated that $55 \%$ of participated mothers scored above the mean score of food hygiene practices. Mothers of children aged 12-23 months had higher odds to practice good food hygiene measures than those of aged 6-11 months [AOR, 1.82 95\% Cl $(1.21,2.73)]$. Mothers whose children attended growth monitoring session practice good food hygiene than their counterpart [AOR, $2.7495 \% \mathrm{Cl}$ $(1.49,5.06)]$. Mothers having media exposure, and having knowledge on critical times of hand washing had relatively good food hygiene measures [AOR, $0.7395 \mathrm{Cl}(1.14,2.62)$ ]. Among food hygiene measures; handwashing with water and soap were low before eating food (17\%), before feeding children (21\%) and before preparing food (32\%) when compared with after visiting the toilet (81\%) and touching dirt (68\%).

Conclusion and recommendation: The study identified that food hygiene practices in the current study were mainly associated with child age, growth monitoring follow-up, maternal awareness about critical times of hand washing, and media exposure. So, improving knowledge of mothers on critical times of handwashing, strengthening growth monitoring follow-up and media promotion are important measures to improve food hygiene practices among mothers of infants and young children.

\section{Introduction}

Food hygiene is the conditions and measures necessary to ensure the safety of food from production to consumption. Food can become contaminated at any point during slaughtering or harvesting, processing, storage, distribution, transportation and preparation $[1,2]$. Complementary food is a food other than breast milk or infant formula introduced for infants and children aged 6 to 23 months [3]. Appropriate food hygiene practices have been found to reduce the risk of diarrhea in children below 2 years of age [4]. The most critical household food hygiene actions to be practiced by mothers in developing country settings include cooking at adequate temperature, decreasing the time food is stored at room temperature, reheating at adequate temperature, adequate hand washing at all critical times, use of clean 
utensils and storage of food at sufficiently low or high temperatures to prevent bacterial multiplication [1, 5].

The period of complementary feeding, which covers a child from 6-23 months of age, is a very vulnerable period when under nutrition starts in many infants, contributing to the high prevalence of undernutrition in children under two years of age [6]. Under nutrition is the outcome of insufficient food intake to meet the body's requirements for nutrients, and/or poor absorption and/or poor biological use of nutrients consumed as a result of repeated infectious disease $[7,8]$.

Foodborne disease is a problem in both developing and developed countries [1, 5]. In low-resource settings, food given to young children is often highly contaminated with fecal pathogens [7]. The burden of disease due to contaminated food is, therefore, in the African region, causing strain on health care systems; severely affects infants and young children; creates a vicious cycle of diarrhea and malnutrition; and hurts the national economy and development and international trade $[1,9]$. Repeated episodes of diarrhea in the first 2 years of life adversely affect a child's nutritional status [10]. A study revealed that around $72 \%$ of deaths associated with diarrhea happen in this period of life [11].

International consultations among professionals identified hand washing with soap and water as a top priority in all settings [12]. A study confirmed that bacteria were found after no hand washing in $44 \%$ of samples; this figure was reduced to $23 \%$ after washing hands with water alone and to $8 \%$ after washing hands with water and soap [13]. Therefore, maternal hand washing with soap is important to reduce the risk of diarrhea in children [14]. Even though hand washing with soap and water is a simple and efficient method for reducing the risk of infectious diseases [13], studies showed that mothers do not wash their hands regularly $[15,16]$.

World Health Organization (WHO) recommends keeping clean all food contact surfaces and equipment used in food preparation $[1,17]$. In addition, cooked food should be stored carefully at an appropriate temperature. It should either be kept cold, ideally in a refrigerator (temperature below $5 \circ \mathrm{c}$ ), or it should be kept hot (above 60॰c) $[1,18]$. Storage of prepared food at room temperature for a long period of time for multiple feeding is the main factor for contamination of complementary food $[19,20]$. A study indicated that a load of microorganisms were high in complementary foods which stayed at room temperature for four hours as compared with the counts taken immediately after preparing the food [20]. For this reason, WHO recommends that food should be eaten immediately within 2 hours or stored food should be reheated thoroughly before feeding $[1,17]$. Even though reheating is vital in the prevention of foodborne disease, studies indicate that reheating of leftover foods fed to infants is rarely practiced $[19,21]$. This study was aimed to assess complementary food hygiene practices among mothers of children aged 6-23 months in Robe town, South East Ethiopia.

\section{Methodology}

A community based cross-sectional study was conducted from March to April 2017 in Robe town, Southeast Ethiopia. The source populations of the study were all mothers of infants and young children 
aged 6-23 months who live in the town. The study population was all sampled mothers of children aged 6-23 months. Mothers who are permanent residents (resides in the town at least for 6 months) and have infants or young children aged 6-23 months were included in the study.

The sample size was determined by using Epi Info version 7 statistical sample size calculator for a population survey. The systematic sampling technique was employed to select the study participants. Data were collected using an interviewer-administered and structured questionnaire. The data collection tools regarding the various socio-demographic and environmental variables were adopted from Ethiopian Demographic and Health Survey (EDHS) questionnaire with some modification to fit with the context [22]. A data collection tool for food hygiene practices were adapted from FAO guidelines for assessing nutrition-related Knowledge, Attitude and Practice (KAP) [23] and some food hygiene questions were developed from WHO Infant and Young Child Feeding (IYCF) guideline [17]. The questionnaire was prepared in English and translated into the mother tongue of the study population. It was pre-tested and its validity and reliability checked before actual data collection time.

The data were collected using pre-coded questions that relate to food hygiene practices by interviewing the mothers and questions on data of household sanitation facilities like latrine type was accompanied with observation. Data were collected by six personnel, one degree, and five diploma nurses. Data collectors were supervised by two public health professionals. Supervisors and data collectors were trained in the overall introduction of the study process. During data collection, the supervisors reviewed and monitored every questionnaire for completeness, clarity, and consistency. The collected data were checked every day by the investigator and correction feedback was given every morning for supervisors and data collectors.

Statistical analysis was performed using SPSS version 20. Descriptive statistics were used to describe the frequency and percentage of each variable. Bivariable logistic regression was used to check which variables had an association with the dependent variable individually and multivariable logistic regression was conducted to identify factors that were associated with complementary food hygiene practices. All variables with $p$-value $<0.25$ in bivariate logistic regression were moved into multivariable logistic regression. Then, independent predictors were determined to be predictive for outcome variable using $p$-value $<0.05$. Adjusted odds ratios with $95 \%$ confidence interval were used to write reports of the analysis.

\section{Operational definitions}

Food hygiene practices: To assess the level of food hygiene practices, mothers were asked food hygiene practice questions from the questionnaire and those who had scored less than the mean value were considered as having poor food hygiene practices and those who scored greater than the mean value were considered as having good food hygiene practices [4]. 
Knowledge on critical times of hand washing: To assess the level of knowledge on key moments for hand washing, mothers were asked one question that has a list of six correct answers. Each respondent is given a score based on the number of correct responses provided. Those mothers who scored less than the mean score were considered as not having knowledge and those who mentions greater than the mean score were considered as having knowledge on critical times of hand washing [23].

\section{Results}

\section{Food hygiene practices}

\section{Food preparation and storage practice}

Table 1 shows the complementary food hygiene practices among mothers/ caregivers in Robe town, Southeast Ethiopia. Four hundred sixty-seven mothers, 91.9\% cook food for their child separately from food of other family members, while the rest mothers reported they were given food for their children from domestic foods prepared for other family members. This study also revealed that $30.5 \%$ and $40.2 \%$ of mothers reported that prepared food is served immediately after preparation and within two hours, respectively. But, the study revealed that $29.3 \%$ mothers were storing prepared food for more than two hours, which they use to serve it two and more times. Four hundred sixty-six mothers, $87.8 \%$ store prepared food in a covered place. However, $9.6 \%$ of interviewed mothers store uncovered food in room temperature. Forty-one $8 \%$ of participated mothers store food in the refrigerator and $2 \%$ of them left it as hot in high temperature (most of them responded they left it on the stove which they used for cooking) until served (Table 1).

\section{Reheating practice}

More than half, $58 \%$ of mothers participated in the study reported they always reheat food when they want to give their child ready to eat food (cooked food) that stayed more than two hours at room temperature. Whereas $28 \%$ of the study participants discard it and $13.8 \%$ of them feed without reheat.

\section{Hand washing at critical times}

Concerning hand washing at critical times, most mothers, $81.5 \%$ washed their hands after visiting the toilet and only $32.7 \%$ of them reported they wash their hands before feeding their child. But the proportion of mothers who have practiced hand washing with soap at a critical time was lower. For instance, $17.5 \%$, $21.3 \%$ and $32.7 \%$ of them were reported, they wash their hands with soap before eating food, before feeding baby and before preparing food, respectively (Figure 1). Most of the mothers who did not report hand washing with soap at critical times reported that they use soap other times such as; after they eat or prepare foods containing fat and oil, after touching charcoal, after the meal, morning and evening, and before religious practices (i.e. Salat). 


\section{Method of child feeding}

The mothers were asked type of utensils or methods they use to feed their child. According to the result of the study, $86.6 \%$ of mothers feed with a spoon/cup, and $40.7 \%$ of them use their bare hands. From which $28.5 \%$ of mothers use both spoon and bare hand simultaneously to feed depending on types of food. For foods like bread and 'injera' they use their bare hands, whereas for foods like milk and semi-solids they use spoon or cups. Bottle feeding was also practiced by $35 \%$ of mothers (Figure 2).

\section{Utensils cleaning practice}

The majority of mothers, $79.7 \%$ and $55.5 \%$ use soap and hot water respectively to clean utensils used for child feeding, whereas some mothers, $20.5 \%$ used only cold water to clean utensils, while few numbers of mothers, $9 \%$ did not use soap and hot water at the same time to clean and sanitize spoons and plates used to feed their children (Table 1).

\section{Domestic waste disposal practices}

Pertaining waste disposal practices, $15 \%$ of mothers dispose of the children's feces to open field. Few mothers responded that they do not dispose child feces in the latrine; they throw out at other places; for example, near the fence where the adults' feet could not reach. In addition, more than half of study participants, $57 \%$ practiced open field disposal of other liquid wastes generating from the household (Table 1).

Table 1: Complementary Food hygiene practices among Mothers or Caregivers in Robe town, Southeast Ethiopia 


\begin{tabular}{|c|c|c|}
\hline Variables & Frequency & $\%$ \\
\hline \multicolumn{3}{|c|}{ Hand washing practice with soap at critical times* } \\
\hline Before food preparation & 166 & 32.7 \\
\hline Before feeding baby & 108 & 21.3 \\
\hline Before eating food & 89 & 17.5 \\
\hline After toilet use & 414 & 81.5 \\
\hline After touching child stool & 347 & 68.3 \\
\hline \multicolumn{3}{|c|}{ Do you wash your child hand before giving food? } \\
\hline Yes & 391 & 77.0 \\
\hline No & 117 & 23.0 \\
\hline \multicolumn{3}{|c|}{ Do you prepare food for the child separately? } \\
\hline Yes & 467 & 91.9 \\
\hline No & 41 & 8.1 \\
\hline \multicolumn{3}{|l|}{ Do you cook child food thoroughly? } \\
\hline Yes & 504 & 99.2 \\
\hline No & 4 & 0.8 \\
\hline \multicolumn{3}{|c|}{ Duration of cooked child meal lasts until served } \\
\hline Immediately after preparation & 155 & 30.5 \\
\hline Less than 2 hours & 204 & 40.2 \\
\hline More than 2 hours & 149 & 29.3 \\
\hline \multicolumn{3}{|l|}{ Where you store child meal?* } \\
\hline In the refrigerator & 41 & 8.1 \\
\hline Kept as hot & 9 & 1.8 \\
\hline Kept in room temperature & 458 & 90.8 \\
\hline Kept covered & 446 & 87.8 \\
\hline \multicolumn{3}{|c|}{ What do you do leftover stayed more than 2 hrs? } \\
\hline Reheat always before feeding & 296 & 58.3 \\
\hline Never/sometimes reheat & 70 & 13.8 \\
\hline Discard & 142 & 28.8 \\
\hline \multicolumn{3}{|c|}{ Which methed do you use to feed your child?* } \\
\hline Feed with spoon & 440 & 86.6 \\
\hline Feed with a bare hand & 207 & 40.7 \\
\hline \multicolumn{3}{|l|}{ Do you practice bottle feed? } \\
\hline Yes & 178 & 35.0 \\
\hline No & 330 & 65.0 \\
\hline \multicolumn{3}{|c|}{ Which method you practice to clean utensils?* } \\
\hline Wash with hot water & 282 & 55.5 \\
\hline Wash with detergent & 405 & 79.7 \\
\hline Wash with cold water only & 104 & 20.5 \\
\hline \multicolumn{3}{|c|}{ Do you wash raw fruits and vegetable before use? } \\
\hline Yes & 490 & 96.5 \\
\hline No & 18 & 3.5 \\
\hline \multicolumn{3}{|l|}{ Child's feces disposal practice } \\
\hline Closed (in latrine) & & \\
\hline
\end{tabular}


Open field

Where you dispose other liquid wastes?

Closed (in the latrine or in sewage pit)

Open field

Notes: *, multiple responses

\section{Factors associated with food hygiene practices}

According to the result of the study the overall food hygiene practice of mothers was significantly associated with child age, growth monitoring follow-up, media exposure and knowledge of mothers on critical times of hand washing. Mothers of children aged 12-23 months practice 1.63 times good food hygiene than that of children aged 6-11 months of age [AOR, $1.8295 \% \mathrm{Cl}(1.21,2.73)]$. Mothers whose child attend growth monitoring session practice good food hygiene than their counterpart [AOR, 2.74 95\% $\mathrm{Cl}(1.49,5.06)]$. The finding also identified that mothers who have media exposure practiced good food hygiene practices compared to mothers who have no media exposure [AOR, $0.7395 \mathrm{Cl}(1.14,2.62)]$. Mothers who have knowledge on critical times of hand washing have more likely to practice better food hygienic practices than mothers who does not have knowledge on critical times of hand washing [AOR, $1.9495 \% \mathrm{Cl}(1.33,2.82)]$. Educational status of mothers, occupation of fathers and marital status of mothers showed significant association in bivariable regression, but not in a multivariable regression model (Table 2).

Table 2: Bivariable and multivariable logistic regression analysis of factors on Complementary food hygiene practice among mothers of children aged 6-23 months in Robe town 


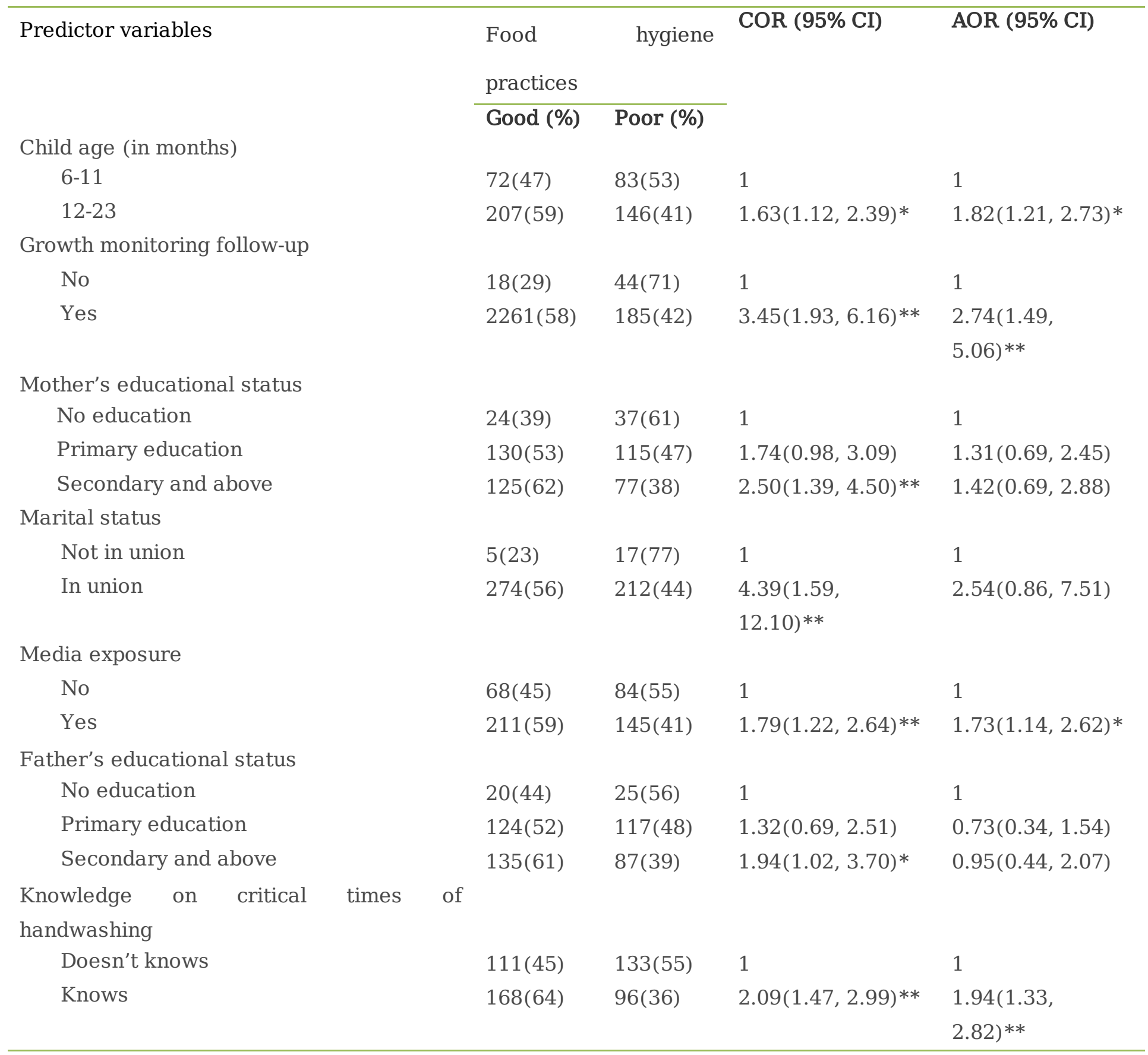

Notes: **, statistically significant at $p<0.01{ }^{*}$, statistically significant at $p<0.05 ;$ COR, Crude odds ratio; AOR,

Adjusted odds ratio; CI, Confidence interval

\section{Discussion}

The result showed that $55 \%$ of interviewed mothers scored above the mean of overall food hygiene practices. Their practice of food hygiene is significantly associated with age of the child. Mothers of younger children had lower odds of practicing good complementary food hygiene than those of older children (12-23 months). This might be because most of the time mothers consider hygiene measures for 
their children when they become older, particularly hand washing for infants were not common. Mothers having media exposure and knowledge on critical times of hand washing practice relatively good food hygiene, compared to their counterparts. This confirms the reality that media exposure has a positive association with their knowledge, which in turn has a positive influence on their practice of good food hygiene. In addition, mothers whose child attended growth monitoring session practice good food hygiene than those who did not attend. This might be as a result of counseling given to mothers or caregivers during this session.

When we see handwashing practices of the study participants, the study showed that $33 \%$ of mothers reported they wash their hands with water and soap before food preparation. It was lower than study conducted in India, which was 74\% [24]. This difference might be due to the difference in the study area and period. Handwashing practice with water and soap after using latrine was $81 \%$, which was better, however, only $21 \%$ of mothers reported they wash their hands with water and soap before feeding their children. This finding was low compared to that of India $79 \%$ and Bangladesh $55 \%[15,24]$. Some of the mothers reported that they wash their hands with soap only during special occasions, which are not considered as critical times like after eating foods containing fat or oil, the morning when they wake up and when they touch like fumes and charcoal wich have minimal public health importance. These mothers did not have a habit of washing their hands with soap at the critical times to prevent diseases, which could be risk for children's and other families' health.

Concerning cleaning and sanitizing of utensils, few mothers were not properly washed the plates and spoons used for serving food after previous use. This problem might be arises from different reasons, like the shortage of water, mothers' workload and inadequate knowledge on the importance of cleaning and sanitizing utensils. They use only cold water to clean utensils, and this may contribute to post cooking contamination of the complimentary food. Ideally, all mothers should clean and sanitize utensils used for an infant or young child's food thoroughly to remove microorganisms found on it. In other way, a number of study participants use bottles with teats to feed their children, which is difficult to clean and sanitize regularly in the context of current study setting. The use of bottles is an obviously harmful practice which increases the risk of food contamination and diarrheal diseases and should not be practiced at all.

The study showed that $40 \%$ of mothers use their bare hand to feed the children. Of which only $19 \%$ of them wash their hands using soap and water, which also contributes to the contamination of complementary foods and in turn childhood illnesses $[17,18]$.

According to this finding, $29 \%$ of mothers store prepared child meal for more than two hours to serve it two times and more. The place where this food stored determines the level of contamination. If it refrigerated the bacterial multiplication becomes very slow. If it does not refrigerated it should be eaten soon, no more than 2 hours, before microorganisms have time to multiply. However, the study shown that only $8 \%$ of families store food in the refrigerator. Cooking small amounts of food which child can consume within a short period of time to avoid long periods of storage and spoilage is the first one choice and could solve the problems related to food storage practice especially in low-income families. 
Because there is the issue of lack of proper operation and maintenance of refrigerator, discontinuity in power supply or faulty of temperature control, it could be harmful for the food spoilage and contamination.

This study has shown that $30 \%$ of mothers feed always fresh food immediately after preparation and $29 \%$ of mothers store food for more than two hours to serve it for another time. This can increase the risk of contamination and diarrheal diseases among children due to the fact that microbial counts increased as food stored at room temperature and can reach its infective dose within two hours $[20,25]$. Most of the mother's store foods covered and few of them; however, stored in uncovered place and increase the level of food contamination [26]. Ideally, it is important to never store complementary food to prevent contamination [27]. But, especially in low socioeconomic households whose purchasing power is low, it is difficult to discard leftovers at every time the food is prepared for the children. Thus, it is better to educate mothers the principles of food hygiene practices.

The study also revealed that $58 \%$ of mothers always use the reheating method after serving left over.

Reheating is easy to prescribe, but could be difficult, especially for poor and busy mothers to practice. The reason for reheating food among our mothers is another critical issue to be considered, because most of them practice reheating simply to make foods warm to make it palatable, rather than to destroy harmful microorganisms. As previous studies show, reheating foods before consumption can considerably reduce food contamination and the risk of illness $[25,28]$.

The mothers' food hygiene practices were determined based on self-reported data which might introduce social desirability bias; and it could overestimate the appropriate practices. This limitation was considered in the study procedures while interviewing the mothers to minimize it as much as possible.

\section{Conclusion}

Food hygiene practices score of mothers was affected by factors like child age and media exposure. Usual handwashing practice with water and soap at all critical times were relatively low before eating food, before feeding children and before preparing food. The practice of food storage at an appropriate temperature was also low. Unhygienic child feeding methods, like bottle feeding and feeding by bare hand, are the identified undesirable food hygiene practices in the current study area.

The findings suggest the following recommendation for effective intervention strategies of infants and child feeding practices.

- Urban health care workers and other health care staffs need to educate mothers on how they improve hygienic handling practices, especially for infants under one year of age.

- Health care workers need to strengthen counseling mothers during growth monitoring sessions on complementary food handling.

\section{Declarations}




\section{Conflicts of interest}

The authors declare that there is no conflict of interest.

\section{Ethical approval and consent}

Ethical issues were considered during the study. A letter of permission and ethical clearance were obtained from Madda Walabu University. The privacy and confidentiality of information from each respondent were maintained through anonymity. Informed written consent of each respondent was secured before the commencement of the interviews. The mothers were given the freedom to refuse or participate in information provisions.

\section{Authors' contributions}

Conceptualization: SB was generated the research area and developed the proposal, and performed data analysis and prepared the original manuscript: TB, MT were involved in proposal development, data analyses and reviewed the research paper. All authors read and approved the final manuscript.

\section{Acknowledgment}

We thank Madda Walabu University and town administration for providing permission, and data collectors, supervisors and study participants for their participation in the study.

\section{References}

1. World Health Organization, Five keys to safer food manual, Department of Food Safety, Zoonoses and Foodborne Diseases: World Health Organization: Avenue Appia $20 \mathrm{CH}-1211$ Geneva 27Switzerland, 2006.

2. World Health Organization, Campaign tool kit, World Health Day 2015 - Food Safety: Campaign at a glance, 2015.

3. World Health Organization, Infant and Young Child Feeding Model Chapter for Textbooks for Medical Students and Allied Health Professionals, World Health Organization, Geneva, Switzerland, 2009

4. Agustina R, Sari TP, Satroamidjojo S, Ingeborg MJ, et al., Association of food-hygiene practices and diarrhea prevalence among Indonesian young children from low socioeconomic urban areas, BMC Public Health, vol. 13, 977, 2012.

5. Woldt M and Moy GG, Literature Review on Effective Food Hygiene Interventions for Households in Developing Countries, 2015. 
6. Food and Agricultural Organization, Complementary Feeding for Children Aged 6-23 Months: A Recipe Book for Mothers and Caregivers, Food and Agriculture Organization of the United Nations (FAO): Street 370, Boeng Kengkang 1, Khan Chamcar Morn Phnom Penh, Cambodia, 2011.

7. World Health Organization, Improving Nutrition Outcomes with Better Water, Sanitation and Hygiene: Practical Solutions For Policies And Programmes, 2015.

8. Food and Agricultural Organization, Regional overview of food insecurity: African food security prospects brighter than ever, Food and Agriculture Organization of the United Nations (FAO): Accra, 2015.

9. World Health Organization, Estimates of the global burden of foodborne diseases, Foodborne Disease Burden Epidemiology Reference Group 2007-2015: 20 Avenue Appia, 1211 Geneva 27, Switzerland, 1-255, 2015.

10. Checkley W, Buckley G, Gilman RH, Assis AM, et al., Multi-country analysis of the effects of diarrhoea on childhood stunting, International Journal of Epidemiology, vol. 37, no. 4, 816-830, 2008. doi:10.1093/ije/dyn099.

11. Walker C, Rudan I, Liu L, Nair H, et al., Global burden of childhood pneumonia and diarrhoea, Lancet, 381, 1405-16, 2013.

12. WHO/UNICEF, Progress on Drinking Water, Sanitation and Hygiene: 2017 Update and SDG Baselines, World Health Organization (WHO) and the United Nations Children's Fund (UNICEF). Licence: CC BYNC-SA 3.0 IGO: Geneva, 2017.

13. Burton M, Cobb E, Donachie P, Judah G, et al., The Effect of Handwashing with Water or Soap on Bacterial Contamination of Hands, International Journal of Environmental Research and Public Health, vol. 8, 97-104, 2011. doi:10.3390/ijerph8010097.

14. Aluisio AR, Maroof Z, Chandramohan D, Bruce J, et al., Risk Factors Associated with Recurrent Diarrheal IIInesses among Children in Kabul, Afghanistan: A Prospective Cohort Study, PLoS ONE, vol. 10, no. 2, 2015. doi:10.1371/journal.pone.0116342.

15. Nizame FA, Unicomb $\mathrm{T}$, Sanghvi $\mathrm{S}$, Roy M, et al., Handwashing before Food Preparation and Child Feeding: A Missed Opportunity for Hygiene Promotion, The American Journal of Tropical Medicine and Hygiene, vol. 89, no. 6, 1179-1185, 2013.

16. Takanashi K, Chonan Y, Quyen DT, Khan NC, et al., Survey of food-hygiene practices at home and childhood diarrhoea in Hanoi, Viet Nam, Journal of Health, Population and Nutrition, vol. 27, no. 5, 602-611, 2009.

17. World Health Organization, Infant and young child feeding Model Chapter for textbooks for medical students and allied health professionals: Geneva Switzerland, 2009.

18. Federal Ministry of Health, Urban Health Extension Program Integrated Refresher Training Module Three; Water, Sanitation and Hygiene Facilitator's Guide, Federal Ministry of Health: Addis Ababa, Ethiopia, 2016.

19. Kung'u JK, Boor KJ, Ame SM, Ali NS, et al., Bacterial Populations in Complementary Foods and Drinking-water in Households with Children Aged 10-15 Months in Zanzibar, Tanzania, Journal of 
Health, Population and Nutrition, vol. 27, no. 1, 41-52, 2009.

20. Islam MA, Ahmed T, Faruque ASG, Rahman S, et al., Microbiological quality of complementary foods and its association with diarrhoeal morbidity and nutritional status of Bangladeshi children, European Journal of Clinical Nutrition, vol. 66, 1242-1246, 2012.

21. Saleh F, Ara F, Hoque MA, and Alam MS, Complementary Feeding Practices among Mothers in Selected Slums of Dhaka City: A Descriptive Study, Journal of Health, Population and Nutrition, vol. 32 , no. 1, 89-96, 2014.

22. Central Statistical Agency (Ethiopia) and ICF International, Ethiopia Demographic and Health Survey 2011. Addis Ababa, Ethiopia and Calverton, Maryland, USA, 2012.

23. Macías YF and Glasauer P, Guidelines for assessing nutrition-related knowledge, attitudes and practices - KAP Manual, Food and Agriculture Organization of the United Nations: Rome, 2014.

24. Khan S, Kumar V, Priya N, and Yadav SS, Hand washing practices among the caregivers of under five children in rural and urban areas of Moradabad, India: a community based study, International Journal of Medical Science and Public Health, vol. 6, no. 1, 133-138, 2016.

10.5455/ijmsph.2017.10072016573.

25. Omemu AM, Atanda OO, Ayinde IA, and Henshaw FO, Perceptions of mothers on food safety related and the microbiological contamination of complimentary foods-A case study in 2 rural areas in Southwestern Nigeria., Researcher, vol. 3, no. 6, 60-67, 2011.

26. Parvez SM, Kwong L, Rahman MJ, Ercumen A, et al., Escherichia coli contamination of child complementary foods and association with domestic hygiene in rural Bangladesh, Tropical Medicine and International Health, vol. 22, no. 5, 547-557, 2017. doi: 10.1111/tmi.12849. Epub 2017 Feb 24.

27. Bourne LT, Pilime N, Sambo M , and Behr A, Food hygiene and sanitation in infants and young children: a paediatric food-based dietary guideline: a literature review, South African Journal of Clinical Nutrition, vol. 26, no. 3, no. 156-164, 2013.

28. Islam MS, Mahmud ZH, Gope PS, Zaman RU, et al., Hygiene intervention reduces contamination of weaning food in Bangladesh, Tropical Medicine and International Health, vol.18, no. 3, 250-258., 2013.

\section{Figures}

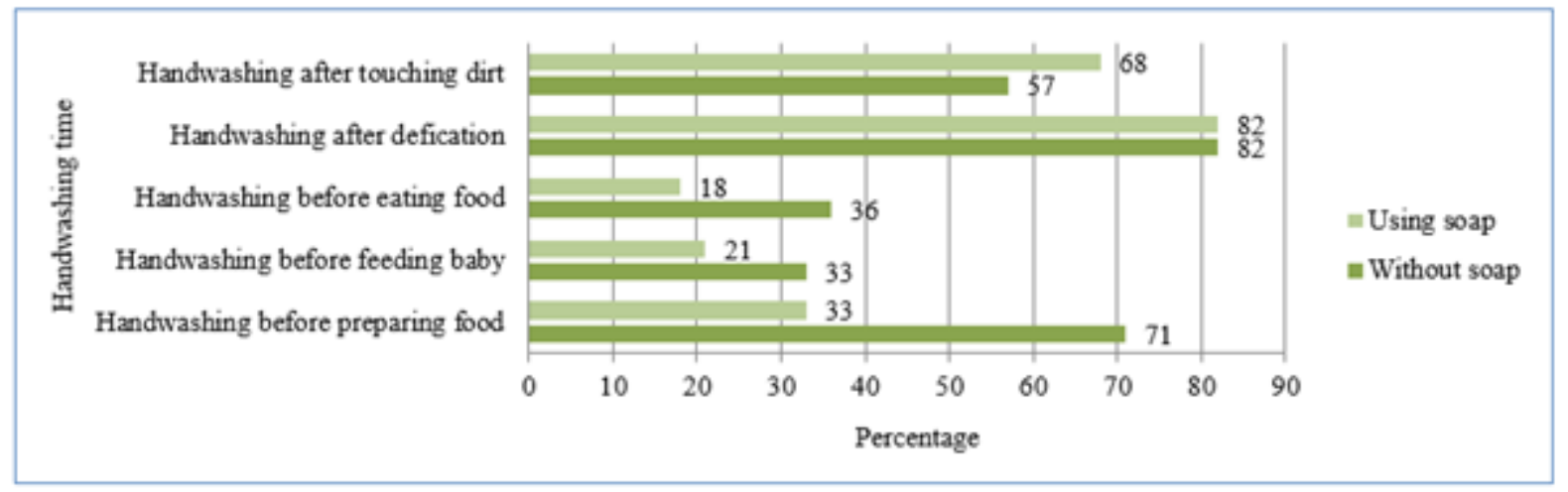

Page $14 / 15$ 
Figure 1

Hand washing practices at critical times with and without using soap among mothers or caregivers in Robe town, Southeast Ethiopia.

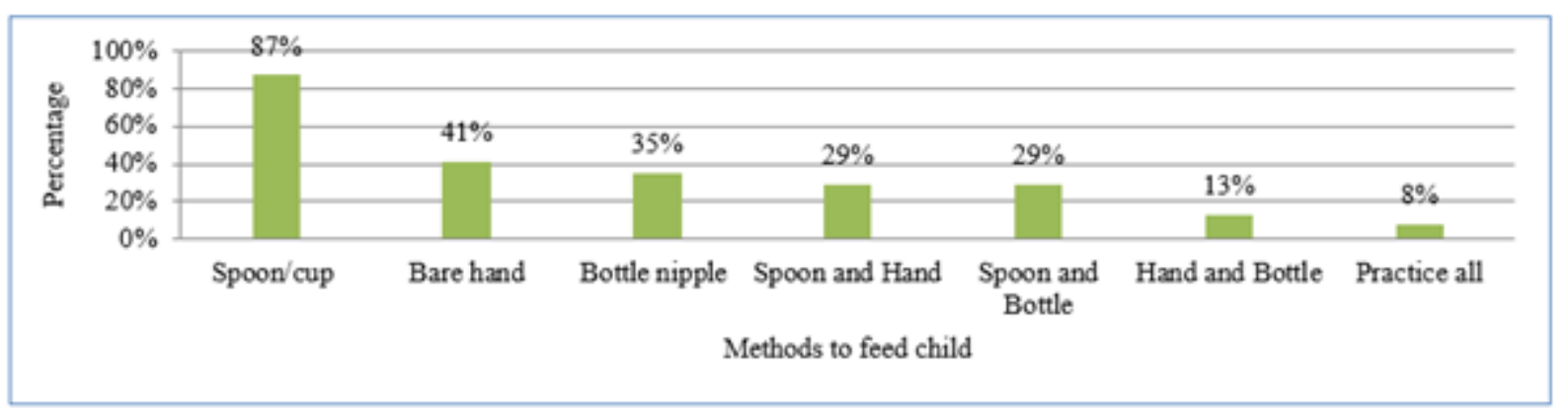

\section{Figure 2}

Methods used to feed their child among mothers of infants and children aged 6-23 months in Robe town, Southeast Ethiopia. 